\title{
Quantitative analysis of mitoflash excited by femtosecond laser
}

Shaoyang Wang

Minglie $\mathrm{Hu}$

$\mathrm{Hao} \mathrm{He}$ 


\title{
Quantitative analysis of mitoflash excited by femtosecond laser
}

\author{
Shaoyang Wang, ${ }^{a}$ Minglie $\mathrm{Hu}^{\mathrm{a}}$ and $\mathrm{HaO} \mathrm{He}^{\mathrm{a}, \mathrm{b}, *}$ \\ ${ }^{a}$ Tianjin University, College of Precision Instrument and Optoelectronics Engineering, Ultrafast Laser Laboratory, Tianjin, China \\ bShanghai Jiao Tong University, School of Biomedical Engineering, Shanghai, China
}

\begin{abstract}
Mitochondrial oxidative flashes (mitoflashes) are oxidative burst events in mitochondria. It is crosslinked with numerous mitochondrial molecular processes and related with pivotal cell functions such as apoptosis and respiration. In previous research, mitoflashes were found as spontaneous occasional events. It would be observed more frequently if cells were treated with proapoptotic chemicals. We show that multiple mitoflashes can be initiated by a single femtosecond-laser stimulation that was tightly focused on a diffraction-limited spot in the mitochondrial tubular structure. The mitoflash events triggered by different photostimulations are quantified and analyzed. The width and amplitude of mitoflashes are found very sensitive to stimulation parameters including laser power, exposure duration, and total incident laser energy. This study provides a quantitative investigation on the photostimulated mitoflashes. It may thus demonstrate such optical method to be a promising technique in future mitochondrial research. @ 2018 Society of Photo-Optical Instrumentation Engineers (SPIE) [DOI: 10.1117/1.JBO .23.6.065005]
\end{abstract}

Keywords: mitoflash; mitochondria; femtosecond laser.

Paper 180134RR received Mar. 6, 2018; accepted for publication Jun. 13, 2018; published online Jun. 27, 2018.

\section{Introduction}

Mitochondrial oxidative flashes (mitoflashes) are oxidative bursts in mitochondria that root from complex mitochondrial molecular dynamics. Over the last decade, research has unveiled that mitoflash generation depends on mitochondrial respiratory function and is controlled by transient opening of mitochondrial permeability transition pore (mPTP). ${ }^{1}$ The activity of mitoflash is highly related with cellular metabolic states ${ }^{1-6}$ (especially mitochondrial energy metabolic behaviors). Mitoflash has also been ubiquitously observed in diverse biological systems such as isolated mitochondria, cells in culture dishes, explanted organs, and enormous models ranging from worms and zebrafish to rodents and humans. ${ }^{1,5-8}$ Thus it was suggested as an elemental signaling event ${ }^{2,9}$ in a variety of physiological and pathophysiological conditions.

Mitoflashes are related with various molecular dynamics. It can be visualized by diverse biosensors or chemical probes which are sensitive to superoxide or other reactive oxygen species (ROS), redox potential or matrix $\mathrm{pH} .{ }^{1-3,10}$ The fluorescent signals of mitoflashes were observed as transient pulses, indicating the bursts of oxidative molecules. Such transient oxidative bursts inside mitochondria are usually associated with other processes including mitochondrial membrane potential depolarization $^{1}$ and transient oxidation of NADH and $\mathrm{FADH}_{2}{ }^{2,4}$ Generally, a spontaneous mitoflash event starts from a stochastic, flickering opening of mPTP triggered by matrix $\mathrm{Ca}^{2+}, \mathrm{ROS}, \mathrm{H}^{+}$, or other factors, which then results in ionic fluxing and the inner mitochondrial membrane (IMM) depolarizing. The electron flow path is then disturbed to increase electron leakage and oxidative stress at intermediary electron transport chain (ETC) sites. Finally, the burst production of superoxide or ROS derivatives can be found. Meanwhile, depolarization of IMM also accelerates electron transfer from the donor pool to ETC acceptors (oxidation of NADH or $\mathrm{FADH}_{2}$ ) and shifts redox state toward oxidation. All those changes induce alkalinization in the matrix simultaneously. Throughout the interconnected mitochondrial network, the membrane depolarization may trigger additional mPTP opening and lead to multiple synchronous (both temporal and spatial) mitoflash activities. ${ }^{1-4,10}$ There are multiple methods, such as inhibiting the $\mathrm{mPTP}$ opening or dissipating membrane potential and $\mathrm{pH}$ gradients across the IMM,,$^{1,2,4}$ to abrogate the mitoflash activities. The mitoflash occurrence can also be effectively blocked in the absence of ETC activities. ${ }^{1-4}$

Foregoing studies have also shown that mitoflash took an important part in multitudinous cell functions. It has been found participating in synaptic plasticity of hippocampal neurons. ${ }^{11}$ The frequency of mitoflash modulates ATP homeostasis in heart cells ${ }^{12}$ and affects somatic cell reprogramming. ${ }^{13}$ It can negatively regulate neural progenitor cell differentiation and proliferation. ${ }^{14}$ Furthermore, acting as a digital reporter of aging process, the frequency of mitoflashes can predict the lifespan of Caenorhabditis elegans in early adulthood. ${ }^{7}$ The spontaneous mitoflash generation is believed to be regulated by physiological factors including mitochondrial basal ROS level, matrix $\mathrm{Ca}^{2+}$, and protons in the IMM. ${ }^{1,15-17}$

In previous studies, mitoflashes were usually observed by chance when treating cells with chemicals to provide indirect stress to mitochondria., ${ }^{1,5,18}$ Hence, the mitoflash events cannot be acquired at predesigned position or time spot. In this regard, it is challengeable and ponderable to develop a controllable method to elicit mitoflash at single-mitochondrion level in targeted living cells. Recently, we have reported the simulation 
by the femtosecond laser (fs-laser) could excite mitoflash at single mitochondrial tubular level in a controllable and precise manner. ${ }^{19,20}$ It was soon used to investigate the occurrence of mitoflashes in the context of structural long-term potentiation at hippocampal synapses. ${ }^{11}$

However, in molecular biological research, this photostimulation method still needs further quantitative investigation. In this report, we will quantitatively show and analyze the fluorescent-indicated multitime mitoflashes triggered by single fs-laser stimulation. The mitoflash fluorescence signals will be depicted by parameters including response time, pulse width, amplitude, mitoflash numbers, and time interval between mitoflashes. Correspondingly, the width and amplitude of the mitoflashes can also indicate different photostimulations that the mitochondria experienced. Our research provides a deeper understanding and statistic results on mitoflash signaling, mitochondrial metabolism, and homeostasis regulation.

\section{Methods}

\subsection{Cell Culture and mt-cpYFP Transfection}

HeLa cells were used as the proof of principle demonstration in this study. Cells were cultured in Dulbecco's modified Eagle's medium (DMEM) supplemented with $10 \%$ fetal bovine serum and incubated at $37^{\circ} \mathrm{C}$ with $5 \% \mathrm{CO}_{2}$. In experiments, cells were seeded in 35-mm Petri dishes with a 0.17-mm glass slide (Nest) for confocal microscopy and photostimulation. Mitochondrial matrix-targeting circularly permuted yellow fluorescent protein (mt-cpYFP) was used as a mitoflash biosensor. ${ }^{1}$ Cells were transfected with mt-cpYFP plasmid at a concentration of
$2.5 \mu \mathrm{g} / \mathrm{mL}$ by polyethylenimine (PEI) $(1 \mathrm{mg} / \mathrm{mL})$. The required amount of plasmid was dissolved in $100 \mu \mathrm{L}$ DMEM, and then PEI was added in a volume to achieve PEI/DNA ratio at 5/1. The PEI-plasmid mixture was incubated at room temperature for $10 \mathrm{~min}$ and added to cell culture medium drop by drop. After transfection, cells were incubated for $24 \mathrm{~h}$ before experiments.

\subsection{Photostimulation and Confocal Microscopy}

The photostimulation scheme, which enabled real-time observation and stimulation, was based on a confocal microscope (Olympus FV1000/IX81) coupled with a homemade Ti: Sapphire laser system $(810 \mathrm{~nm}, 75 \mathrm{fs}$, and $80 \mathrm{MHz})$ as shown in Fig. 1(a). The fs-laser was collimated by a lens pair, directly reflected by a beam splitter $(\mathrm{BS}$, reflection $=70 \%)$ to the back aperture of objective lens (Olympus, water immersion, 60×, $\mathrm{NA}=1.2$ ), which could be focused at a single mitochondrial tubule. A dichroic mirror (transmission: $>750 \mathrm{~nm}$; reflection: $<750 \mathrm{~nm}$ ) above the BS was used to split the fs-laser and the continuous wave (CW) laser inside the microscope. A CCD camera under the dichroic mirror was adopted for monitoring the focus of fs-laser. By the wild-field imaging of the CCD camera, the diameter of the laser focus was measured $(\sim 1.5 \mu \mathrm{m})$. Therefore, unlike the scanning focus in two-photon microscopes, the focus of fs-laser was a fixed spot in the central position of the field of view (FOV) in this setup. The output power of fs-laser could be tuned by a round continuously variable metallic neutral density filter (Thorlabs). A mechanical shutter (GCI-73, China Daheng Group) was used to control the laser stimulation duration. In the vertical direction (the laser propagation direction

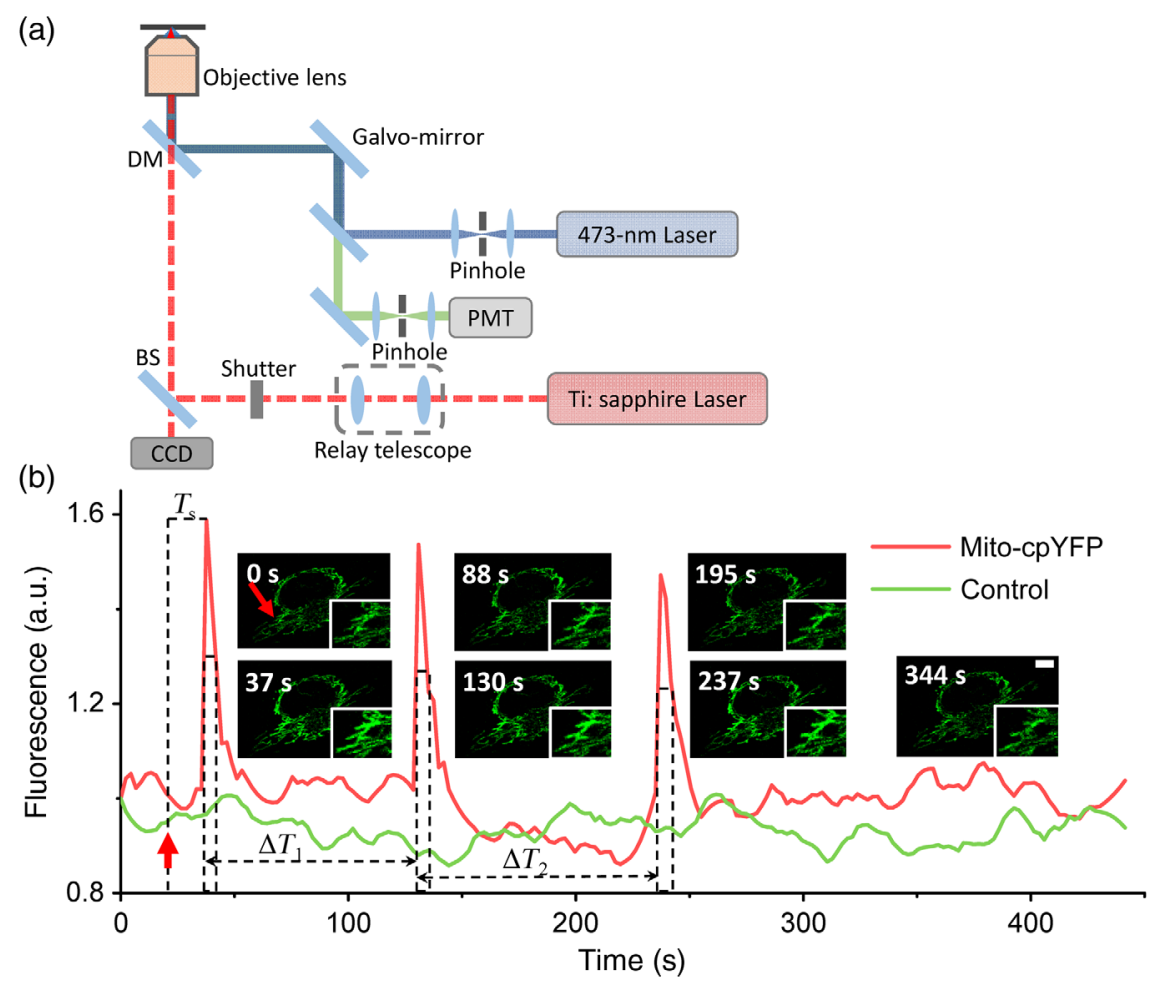

Fig. 1 Photostimulation to mitochondria. (a) The photostimulation scheme based on confocal microscope system. BS, beam splitter; DM, dichroic mirror; and PMT, photomultiplier tube. (b) Multiple mitoflashes acquired from the stimulated mitochondrial tubule. The quantification of the mitoflashes is defined. Red arrow: photostimulation. Insets: time-lapse images of mitoflashes indicated by mt-cpYFP. Bar: $10 \mu \mathrm{m}$. 
vertical to the specimen), the focus of fs-laser was adjusted to the confocal scanning focal plane by tuning distances between the collimating lenses. In this way, any observed mitochondria in the imaging plane could be stimulated by the fs-laser. In experiments, the laser beam was originally cut off by the shutter. Once the targeted mitochondrion was selected, it was moved to the focal position of the fs-laser by adjusting the stage of the microscope (to the center of FOV). Then the shutter would open for a preset duration to deliver photostimulation during $\mathrm{CW}$ laser confocal microscopy (note that the photostimulation would not affect the confocal microscopy). In general, a select cell would be stimulated for only one time, which would excite only one tubular structure of a single mitochondrion inside.

For confocal scanning, mt-cpYFP was excited by a 473-nm laser and detected at 510 to $550 \mathrm{~nm}$. The cells were observed continuously by the confocal microscopy in experiments. In general, for each time the photostimulation was delivered during the confocal imaging, the targeted cell was observed for a total duration of $440 \mathrm{~s}$ to provide an observation window as long as possible to acquire the most mitoflash events while minimizing information missing (such as spontaneous mitoflashes caused by long scanning time of the confocal microscopy). Images were acquired at a speed of one frame $(512 \times 512$ pixels $)$ per $2.2 \mathrm{~s}$.

\subsection{Statistics}

The data were presented as mean \pm standard error of mean (SEM) and analyzed with GraphPad Prism 7 software through the one-tailed paired $t$-test except where stated otherwise.

\section{Results}

\subsection{Mitoflash Excitation}

To manually initiate mitoflashes, mitochondria labeled with mtcpYFP were randomly selected and then stimulated by the fslaser at 16 to $32 \mathrm{~mW}$ (at cell) for 66 to $150 \mathrm{~ms}$. In our experiments, we acquired a very high stimulation-response ratio $(90$ responding mitoflashes from 92 stimulated mitochondria in 92 different cells of 15 independent experiments). However, unlike the randomness of spontaneous mitoflashes, fs-laser initiated mitoflashes were only found in those stimulated mitochondria during the observation period. Generally, around $10 \mathrm{~s}$ after the photostimulation, the stimulated mitochondria showed mitoflash events. As control, we counted the spontaneous mitoflashes in cells without any stimulation. The spontaneous mitoflash events were observed 33 times in total in 262 cells during the 440-s observation duration for each cell.

Intriguingly, multitime mitoflashes could be observed in the stimulated mitochondrial tubule after a single stimulation [Fig. 1(b)]. To describe the properties of mitoflashes in a quantitative manner, we defined parameters as shown in Fig. 1(b). The time interval between the photostimulation (fs-laser exposure) and the first mitoflash peak was defined as the response duration $\left(T_{s}\right)$. The time interval $(\Delta T)$ between two adjacent mitoflashes was defined by the duration between the half peak of the mitoflashes. The time width of each mitoflash was defined by the full duration at half maximum (FDHM). Though the morphology of the mitochondria might change (fragmented and soon recovered or swelled without recovery) ${ }^{19,20}$ after fs-laser stimulation, the actual displacement of the targeted mitochondrion is negligible during the whole observation. Therefore, the statistical fluorescent intensity of the original tubular structure would fully represent the slightly changing mitochondrion.

Fluorescent images [insets in Fig. 1(b)] showed photostimulated mitoflashes in cells labeled with mt-cpYFP. The mitochondrial morphology did not change and the cpYFP fluorescence had no photobleaching after stimulation (stimulation: $16 \mathrm{~mW}$ for $100 \mathrm{~ms}$, observation: $440 \mathrm{~s}$ later). The viability of stimulated cells was validated by the proliferation rate (cell amount afterward/original cell number). To be strict, cells were stimulated at $40 \mathrm{~mW}$ from $100 \mathrm{~ms}$ to $1 \mathrm{~s}$. The proliferation rate was then acquired after 12 and $24 \mathrm{~h}$, respectively (the data of each stimulation time were acquired from five independent tests and presented as mean \pm SEM), as shown in Table 1 . It could be found that the proliferation rate of those stimulated cells was all close to the control (without any stimulation). When the photostimulation duration was increased to $5 \mathrm{~s}$, the proliferation rate decreased dramatically.

The amplitude of a mitoflash, which was defined as the maximum ratio of fluorescence $F$ over the initial baseline $F_{0}\left(F / F_{0}\right)$, was $1.324 \pm 0.012$ ( $n=150$ flashes in 90 stimulated mitochondria) in photostimulated cells, which was close to spontaneous mitoflashes in rat cardiac myocytes $\left(F / F_{0} \sim 1.41 \pm 0.02\right)$, neural progenitor cells $\left(F / F_{0} \sim 1.29 \pm 0.11\right),{ }^{14}$ HeLa cells $\left(F / F_{0} \sim 1.32\right),{ }^{15}$ and in our experiment $\left(F / F_{0} \sim 1.303 \pm\right.$ $0.024)$. This result suggests the mt-cpYFP indication as a stable and universal method for quantitative study of mitoflash.

\subsection{Quantitative Analysis}

In order to quantitatively present this laser-based method, we investigated the properties of mitoflashes excited by photostimulation at different laser powers. With a universal 100-ms duration, we triggered mitoflashes using three sets of moderate power $(16,24$, and $32 \mathrm{~mW}$ at cell) fs-laser stimulation. Each mitochondrion was stimulated for only one single time and thereafter would be observed for $440 \mathrm{~s}$. If at least one-time mitoflash was observed, the excitation would be counted as one effective trial. The efficiency (stimulation-response ratio) of mitoflash excitation was then acquired as $94.44 \%$ at $16 \mathrm{~mW}$ (incident energy: $1.6 \mathrm{~mJ}, n=18$ ), $95.24 \%$ at $24 \mathrm{~mW}$ (incident

Table 1 The proliferation rate of the stimulated cells (at $40 \mathrm{~mW}$ ).

\begin{tabular}{cccccc}
$0.1 \mathrm{~s}(280$ cells & $\begin{array}{c}0.5 \mathrm{~s}(314 \text { cells } \\
\text { in five tests) (\%) }\end{array}$ & $\begin{array}{c}1 \mathrm{~s}(319 \text { cells in } \\
\text { five tests) (\%) }\end{array}$ & $\begin{array}{c}5 \mathrm{~s}(323 \text { cells in } \\
\text { five tests) (\%) }\end{array}$ & $\begin{array}{c}\text { Control (295 cells } \\
\text { in five tests) (\%) }\end{array}$ \\
\hline $12 \mathrm{~h}$ & $136.05 \pm 1.53$ & $135.65 \pm 2.61$ & $138.33 \pm 2.22$ & $118.76 \pm 2.54^{* * *}$ & $141.81 \pm 1.14$ \\
$24 \mathrm{~h}$ & $178.69 \pm 5.27$ & $180.58 \pm 3.65$ & $183.87 \pm 2.98$ & $144.78 \pm 6.55^{* * *}$ & $190.38 \pm 3.87$ \\
\hline
\end{tabular}

${ }^{* * *} P<0.001$ (independent $t$-test). 
energy: $2.4 \mathrm{~mJ}, n=21$ ), and $100 \%$ at $32 \mathrm{~mW}$ (incident energy: $3.2 \mathrm{~mJ}, n=17)$. To check if the mitoflash excitation was energy dependent, we tuned the stimulation duration (exposure time) with a fixed fs-laser power to create varied incident fs-laser energy. The stimulation was performed at $24 \mathrm{~mW}$ for $67 \mathrm{~ms}$ (24 mW-S) and $150 \mathrm{~ms}$ (24 mW-L), respectively, to provide the incident energy at 1.6 and $3.2 \mathrm{~mJ}$, the same energy level as the photostimulation at 16 and $32 \mathrm{~mW}$. In this way or alike, we would be able to compare the mitoflashes at different stimulation powers with the same incident laser energy, and at different energies with the same power.

The photostimulation could activate almost immediate mitoflashes in the stimulated mitochondria. We quantified the response time $T_{s}$ of mitoflash at different stimulation powers. As shown in Fig. 2(a), with an increasing laser power, the response duration $T_{s}$ dropped significantly $(7.29 \pm 1.07 \mathrm{~s}$ at $16 \mathrm{~mW}, n=17 ; 5.22 \pm 0.67 \mathrm{~s}$ at $24 \mathrm{~mW}-\mathrm{S}, \quad n=18$; $4.10 \pm 0.42 \mathrm{~s}$ at $24 \mathrm{~mW}, n=20 ; 3.22 \pm 0.33 \mathrm{~s}$ at $24 \mathrm{~mW}-\mathrm{L}$, $n=18 ; 3.35 \pm 0.56 \mathrm{~s}$ at $32 \mathrm{~mW}, n=17$, respectively). With the ascending stimulation laser power, it would take shorter time to observe the first mitoflash in the stimulated mitochondria. But it should be noted that the minimal $T_{s}$ is around $3.28 \mathrm{~s}$ (with enough-high incident energy).

Once the first fs-laser stimulated mitoflashes were initiated, one or more subsequent mitoflashes could be observed in the following 440-s observation window [Figs. 2(b) and 2(c)]. However, the third mitoflash was relatively rare [Fig. 2(c)]. Interestingly, multiflashes were more likely to be induced by weak stimulations. It was very difficult to observe the third mitoflash at 24 or $32 \mathrm{~mW}$. The average number of mitoflashes in the stimulated mitochondrion observed in the window decreased significantly if the laser power increased to $32 \mathrm{~mW}$ [Fig. 2(b)].
Next, we quantified the amplitude $\left(F / F_{0}\right)$ and duration (FDHM) of the multiple mitoflashes excited by a single-time photostimulation as shown in Figs. 2(c) and 2(d). On weak stimulation (16 mW and $24 \mathrm{~mW}-\mathrm{S}$ ) conditions, the amplitude of the first and second mitoflashes was the same $(1.346 \pm 0.031$, $n=17$ and $1.345 \pm 0.052, n=12$ at $16 \mathrm{~mW} ; 1.36 \pm 0.042$, $n=18$ and $1.355 \pm 0.058, n=10$ at $24 \mathrm{~mW}-\mathrm{S})$. The third one showed a little decrease at $16 \mathrm{~mW}(1.275 \pm 0.069, n=5)$ and no change at $24 \mathrm{~mW}-\mathrm{S}(1.365 \pm 0.062, n=6)$. At $24 \mathrm{~mW}$, the first mitoflash amplitude $(1.348 \pm 0.031, n=20)$ was also quite close to that at $16 \mathrm{~mW}$. But the second flash significantly decreased $[1.217 \pm 0.029, n=10$, Fig. 2(c)]. When facing stronger simulations $(24 \mathrm{~mW}-\mathrm{L}$ and $32 \mathrm{~mW})$, the amplitude of the first mitoflash still remained the same $(1.336 \pm 0.04, n=$ 18 at $24 \mathrm{~mW}-\mathrm{L}$ and $1.336 \pm 0.034, n=17$ at $32 \mathrm{~mW})$. But the second amplitude showed a little decrease at $24 \mathrm{~mW}-\mathrm{L}$ $(1.288 \pm 0.034, n=9)$, and a significant decrease at $32 \mathrm{~mW}$ $(1.174 \pm 0.05, n=5)$ compared with the first or the second mitoflash excited by $16 \mathrm{~mW}$. Moreover, the total number of the second or third mitoflash events under photostimulations at higher laser power also decreased significantly compared with ones at weak stimulation.

The width of mitoflashes, determined by the mPTP modulation, was even more sensitive to different photostimulations. As shown in Fig. 2(d), the width variation of the first mitoflash was much larger compared with the amplitude. Generally, with an ascending fs-laser power, the width of the first mitoflash decreased significantly $(15.35 \pm 1.875 \mathrm{~s}, n=17$ at $16 \mathrm{~mW}$; $14.06 \pm 2.139 \mathrm{~s}, n=18$ at $24 \mathrm{~mW}-\mathrm{S} ; 11.95 \pm 1.206 \mathrm{~s}, n=$ 20 at $24 \mathrm{~mW} ; 10.44 \pm 1.149 \mathrm{~s}, n=18$ at $24 \mathrm{~mW}-\mathrm{L}$; and $9.59 \pm 0.515 \mathrm{~s}, n=17$ at $32 \mathrm{~mW}$, respectively). Under each power circumstances, the width of the second mitoflash all increased dramatically compared with the first one
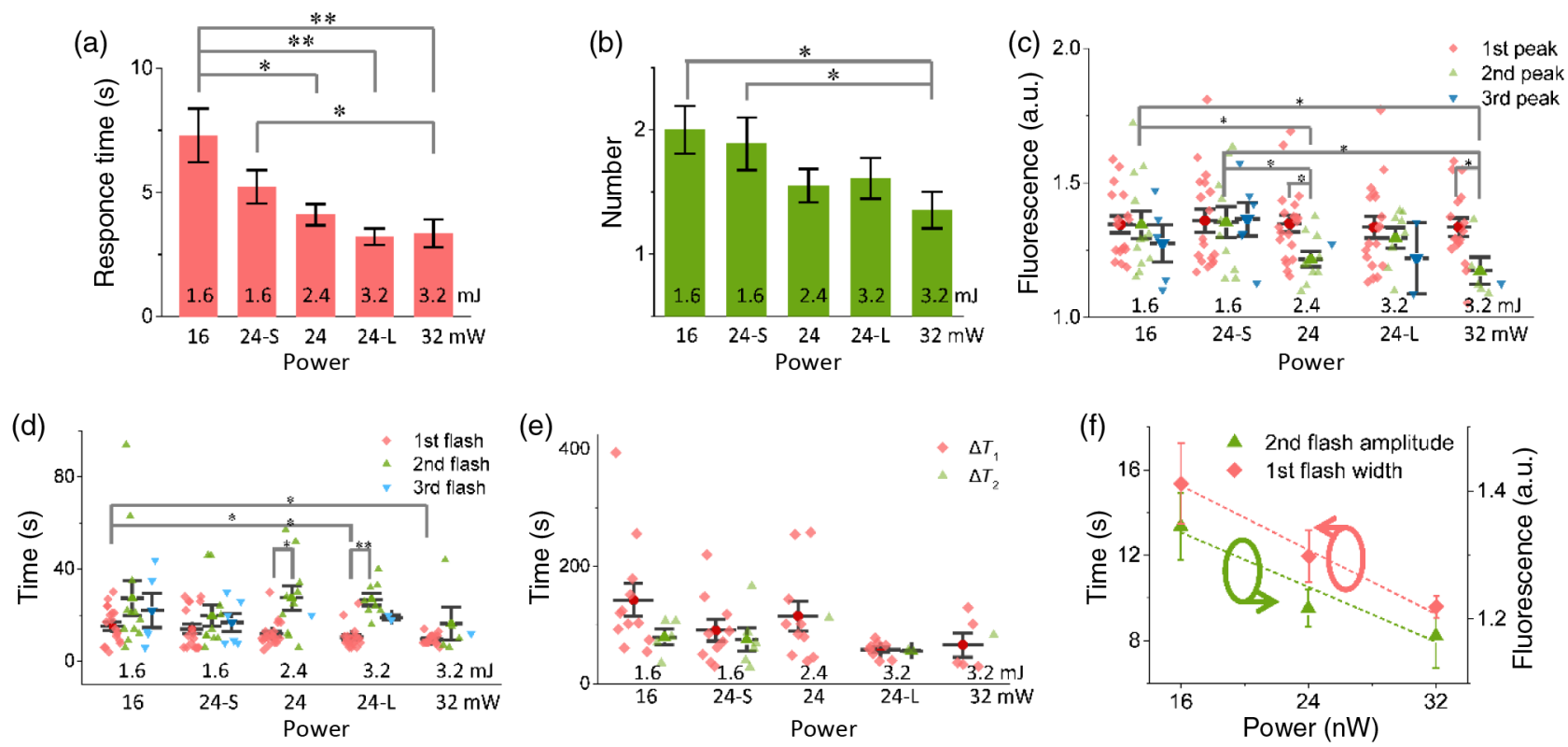

Fig. 2 Quantitative analysis of mitoflashes excited by different laser powers. (a) The response duration of mitoflash could be observed after the photostimulation. (b) The average number of mitoflashes observed in each stimulated mitochondrion. (c) The amplitude and (d) pulse width of each mitoflash. (e) The time interval between multitime mitoflashes observed from a single mitochondrion. (f) The first flash width and the amplitude of second flash were sensitive to different stimulations. $x$-axis label; upper, incident energy level; below, incident power level; 24-S, $24 \mathrm{~mW}$ for $66 \mathrm{~ms}$; and 24-L, $24 \mathrm{~mW}$ for $150 \mathrm{~ms} .{ }^{*} P<0.05$, ${ }^{\star \star} P<0.01$ (independent $t$-test). 
$(27.42 \pm 7.464,19.8 \pm 4.669,27.5 \pm 5.33,25 \pm 3.037$, and $16.4 \pm 7.075 \mathrm{~s}$, respectively).

The time interval $(\Delta T)$ between two adjacent flashes decreased at high-intensity stimulation [Fig. 2(e)]. Notably, at $16 \mathrm{~mW}, \Delta T_{2}$ was shorter than $\Delta T_{1} . \Delta T_{1}$ also decreased along with the increase of stimulation power.

\section{Discussion}

Analyzing the fore-mentioned data, we have found that characteristics of photoactivated mitoflashes such as response durations, amounts, amplitudes, and widths were affected by the photostimulation differentiation. First, according to our results, the instantaneous photostimulation may induce a long-term molecular activity which last from tens to even hundreds of seconds. The resulting mitoflash sequence may indicate the homeostatic dynamics between the optical damage/stimulation and mitochondrial recovery. ${ }^{17}$ The stimulation with higher laser power or longer exposure duration induced more damage to mitochondria. Then mPTP might lose the ability of modulating oxidative stress, resulting in less mitoflashes. The frequency of the mitoflash was supposed to be regulated by mitochondria respiration and transient $\mathrm{mPTP}$ opening. ${ }^{1}$ Hence, the response duration and the amount of excited mitoflashes were both sensitive to the stimulation. Incident fs-laser with higher power/ energy would excite the first mitoflash in a shorter response time but generate less mitoflashes, whereas moderate stimulation at lower power would excite more mitoflashes but with longer response time.

Second, the mitoflash amplitude, determined by the oxidative level in mitochondria, was also related with the photostimulation. At rest, it had the approximate, if not same, oxidative level in all mitochondria. Therefore, the amplitudes of the first flash under different stimulations were close to each other despite the biological variation. However, after the photostimulation, the laser-induced oxidative stress would be different due to the photodamage variations. That is, the severer photostimulation (or photodamage) mitochondrion experienced, the more modulating ability mPTP would lose and the narrower mitoflash amplitude. Such assumption was testified by our results: the amplitude of the first mitoflash $(1.346 \pm 0.031$ at $16 \mathrm{~mW}, 1.36 \pm 0.042$ at $24 \mathrm{~mW}-\mathrm{S}, 1.348 \pm 0.031$ at $24 \mathrm{~mW}$, $1.336 \pm 0.04$ at $24 \mathrm{~mW}-\mathrm{L}$, and $1.336 \pm 0.034$ at $32 \mathrm{~mW}$ ) under different photostimulations was similar and very close to the spontaneous ones (rat cardiac myocyte, $1.41 \pm 0.02{ }^{1}{ }^{\mathrm{n}} \mathrm{neu}-$ ral progenitor cells, $1.29 \pm 0.11 ;{ }^{14}$ HeLa cells, $\sim 1.32 ;{ }^{15}$ and in our experiment, $1.303 \pm 0.024)$. Under the relatively weak stimulations (16 $\mathrm{mW}$ and $24 \mathrm{~mW}-\mathrm{S}$ ), the amplitude of second mitoflash maintained the same while higher power/energy stimulations ( $24 \mathrm{~mW}, 24 \mathrm{~mW}-\mathrm{L}$, and $32 \mathrm{~mW}$ ) induced a significant decrease in amplitude of the second flash.

Third, the mPTP opening duration might be immediately influenced by the photostimulation and such influence could be indicated by the first mitoflash width. In this study, the stimulated mitoflash width at less intense stimulations [15.35 \pm $1.875 \mathrm{~s}(16 \mathrm{~mW}), 14.06 \pm 2.139 \mathrm{~s}(24 \mathrm{~mW}-\mathrm{S}), 11.95 \pm 1.206 \mathrm{~s}$ $(24 \mathrm{~mW})$ at two incident energies, respectively] was close to the spontaneous ones in rat cardiac myocytes $(17.2 \pm 0.44 \mathrm{~s})^{1}$ and neural progenitor cells $(12.06 \pm 1.25 \mathrm{~s}),{ }^{14}$ but much wider than that of HeLa cells $(\sim 9.7 \mathrm{~s}),{ }^{15}$ and the spontaneous ones in our present experiments $(9.97 \pm 0.615 \mathrm{~s})$. Only at high incident energy $[10.44 \pm 1.149 \mathrm{~s} \quad(24 \mathrm{~mW}-\mathrm{L})$ and $9.59 \pm 0.515 \mathrm{~s}$ $(32 \mathrm{~mW})]$, the stimulated mitoflash width was close to the spontaneous ones of HeLa cells. This was probably because the weak photostimulations could initiate excitable-cell-alike processes and thereby exhibited long-duration mitoflashes, whereas strong photostimulations generally damaged the target mitochondrion by photoinduced oxidative stress. Therefore, the photoactivated mitoflash can be applied in mitochondrial metabolism and signaling research. For instance, a previous study showed that photoactivated mitoflash played a relevant role in structural long-term potentiation, the same as nigericin-triggered mitoflashes or spontaneous ones. ${ }^{11}$

We have also found the width of the first mitoflash and the amplitude of the second fitted linearly with the incident fs-laser power, as shown in Fig. 2(f). Hence, those two parameters could serve as an indication of the stimulation (or damage) the mitochondrion experienced, or the mitochondrial wholesomeness.

In addition, it should be noted that the parameters (central wavelength, repetition rate, etc. but not power or exposure time) of the fs-laser may influence the photostimulation effect as well. For example, with a fixed pulse energy, the pulse width could determine the peak power of a pulse and might influence the multiphoton excitation efficiency. The repetition rate provided different heat accumulations at second level exposure. ${ }^{21}$ According to our previous report, ${ }^{18}$ narrow pulse width (high peak power) could induce higher mitoflash excitation efficiency. In contrast, laser thermal effect (mainly dominated by the repetition rate) did minor influence on mitoflash generation.

\section{Conclusion}

In general, photostimulation by the fs-laser provides a controllable and precise way to excite mitoflash in live cells. In this report, we showed that multitime mitoflashes could be activated by a single-time photostimulation focused on a single spot of the mitochondrial tubule. The width of the first mitoflash and amplitude of the second are quite sensitive to different photostimulations. Based on above observations, quantitative analysis was then presented. Such analysis may provide a deeper understanding on fs-laser-induced mitoflash and may contribute to the further development of such high-precision methodology.

\section{Disclosures}

There is no conflict of interest in this work.

\section{Acknowledgments}

We thank Professor Heping Cheng for effective discussions and the gift of mt-cpYFP plasmid. This work was supported by the National Natural Science Foundation of China (NSFC) (Grant Nos. 81661168014, 81571719, and 61535009).

\section{References}

1. W. Wang et al., "Superoxide flashes in single mitochondria," Cell 134, 279-290 (2008).

2. T. Hou et al., "Mitochondrial flashes: new insights into mitochondrial ROS signaling and beyond," J. Physiol. 592(17), 3703-3713 (2014).

3. X. Zhang et al., "Superoxide constitutes a major signal of mitochondrial superoxide flash," Life Sci. 93, 178-186 (2013).

4. L. Wei-LaPierre et al., "Respective contribution of mitochondrial superoxide and $\mathrm{pH}$ to mitochondria-targeted circularly permuted yellow fluorescent protein (mt-cpYFP) flash activity," J. Biol. Chem. 288, 1056710577 (2013)

5. L. Wei et al., "Mitochondrial superoxide flashes: metabolic biomarkers of skeletal muscle activity and disease," FASEB J. 25, 3068-3078 (2011) 
6. H. Fang et al., "Imaging superoxide flash and metabolism-coupled mitochondrial permeability transition in living animals," Cell Res. 21, 1295-1304 (2011).

7. E. Z. Shen et al., "Mitoflash frequency in early adulthood predicts lifespan in Caenorhabditis elegans," Nature 508, 128-132 (2014).

8. M. Zhang et al., "Remodeling of mitochondrial flashes in muscular development and dystrophy in zebrafish," PLOS ONE 10, e0132567 (2015).

9. G. Feng et al., "Mitochondrial flashes: elemental signaling events in eukaryotic cells," in Pharmacology of Mitochondria, H. Singh and S. S. Sheu, Eds., pp. 403-422, Springer, Cham (2016).

10. M. O. Breckwoldt et al., "Multiparametric optical analysis of mitochondrial redox signals during neuronal physiology and pathology in vivo," Nat. Med. 20, 555-560 (2014).

11. Z. Fu et al., "Dendritic mitoflash as a putative signal for stabilizing longterm synaptic plasticity," Nat. Commun. 8, 31 (2017).

12. X. Wang et al., "Mitochondrial flashes regulate ATP homeostasis in the heart," eLife 6, e23908 (2017)

13. Z. Ying et al., "Transient activation of mitoflashes modulates nanog at the early phase of somatic cell reprogramming," Cell Metab. 23, 220226 (2016).

14. Y. Hou et al., "Mitochondrial superoxide production negatively regulates neural progenitor proliferation and cerebral cortical development," Stem Cells 30, 2535-2547 (2012).
15. T. Hou et al., "Synergistic triggering of superoxide flashes by mitochondrial $\mathrm{Ca}^{2+}$ uniport and basal reactive oxygen species elevation," J. Biol. Chem. 288, 4602-4612 (2013).

16. C. Jian et al., "Regulation of superoxide flashes by transient and steady mitochondrial calcium elevations," Sci. China Life Sci. 57, 495-501 (2014).

17. X. Wang et al., "Protons trigger mitochondrial flashes," Biophys. J. 111, 386-394 (2016).

18. W. Shang et al., "Cyclophilin D regulates mitochondrial flashes and metabolism in cardiac myocytes," J. Mol. Cell Cardiol. 91, 63-71 (2016).

19. Y. Wang et al., "Photostimulation by femtosecond laser triggers restorable fragmentation in single mitochondrion," J. Biophotonics 10(2), 286-293 (2017).

20. F. Shi et al., "Mitochondrial swelling and restorable fragmentation stimulated by femtosecond laser," Biomed. Opt. Express 6, 4539-4545 (2015).

21. A. Vogel et al., "Mechanisms of femtosecond laser nanosurgery of cells and tissues," Appl. Phys. B 81(8), 1015-1047 (2005).

Biographies for the authors are not available. 